\title{
Intraoperative subcortical motor evoked potential stimulation: how close is the corticospinal tract?
}

\author{
Ehab Shiban, MD, ${ }^{1}$ Sandro M. Krieg, MD, ${ }^{1}$ Bernhard Haller, PhD, ${ }^{3}$ Niels Buchmann, MD, ${ }^{1}$ \\ Thomas Obermueller, MD, ${ }^{1}$ Tobias Boeckh-Behrens, MD, ${ }^{2}$ Maria Wostrack, MD, ${ }^{1}$ \\ Bernhard Meyer, MD, ${ }^{1}$ and Florian Ringel, MD1
}

\begin{abstract}
1Department of Neurosurgery, and ${ }^{2}$ Section of Neuroradiology, Department of Radiology, Klinikum rechts der Isar, Technische Universität München; and ${ }^{3}$ Institute for Medical Statistics and Epidemiology, Technische Universität München, Munich, Germany
\end{abstract}

\begin{abstract}
OBJECT Subcortical stimulation is a method used to evaluate the distance from the stimulation site to the corticospinal tract (CST) and to decide whether the resection of an adjacent lesion should be terminated to prevent damage to the CST. However, the correlation between stimulation intensity and distance to the CST has not yet been clearly assessed. The objective of this study was to investigate the appropriate correlation between the subcortical stimulation pattern and the distance to the CST.

METHODS Monopolar subcortical motor evoked potential (MEP) mapping was performed in addition to continuous MEP monitoring in 37 consecutive patients with lesions located in motor-eloquent locations. The proximity of the resection cavity to the CST was identified by subcortical MEP mapping. At the end of resection, the point at which an MEP response was still measurable with minimal subcortical MEP intensity was marked with a titanium clip. At this location, different stimulation paradigms were executed with cathodal or anodal stimulation at $0.3-, 0.5-$, and 0.7 -msec pulse durations. Postoperatively, the distance between the CST as defined by postoperative diffusion tensor imaging fiber tracking and the titanium clip was measured. The correlation between this distance and the subcortical MEP electrical charge was calculated.
\end{abstract}

RESULTS Subcortical MEP mapping was successful in all patients. There were no new permanent motor deficits. Transient new postoperative motor deficits were observed in 14\% (5/36) of cases. Gross-total resection was achieved in $75 \%(27 / 36)$ and subtotal resection (> $80 \%$ of tumor mass) in $25 \%$ (9/36) of cases. Stimulation intensity with various pulse durations as well as current intensity was plotted against the measured distance between the CST and the titanium clip on postoperative MRI using diffusion-weighted imaging fiberitracking tractography. Correlational and regression analyses showed a nonlinear correlation between stimulation intensity and the distance to the CST. Cathodal stimulation appeared better suited for subcortical stimulation.

CONCLUSIONS Subcortical MEP mapping is an excellent intraoperative method to determine the distance to the CST during resection of motor-eloquent lesions and is highly capable of further reducing the risk of a new neurological deficit. http://thejns.org/doi/abs/10.3171/2014.10.JNS141289

KEY WORDS intraoperative mapping; corticospinal tract; motor-eloquent lesion; motor evoked potential; diagnostic and operative techniques

$\mathrm{T}$ HE aim of surgery for primary and secondary intrinsic brain tumors is complete tumor resection to improve prognosis and control symptoms, and thereby improve the patient's quality of life. ${ }^{24,39,45,47}$ However, the goal of maximum resection should be met without any new surgery-related neurological deficits arising,,$^{13}$ and all efforts should be made to reduce patients' risk for neurologi- cal damage. Therefore, lesions in or near motor-eloquent regions, such as the precentral gyrus or the corticospinal tract (CST), represent particularly difficult situations in neurosurgery, and precautions need to be taken to prevent postoperative damage while maximizing resection.

Intraoperative electrophysiological mapping and monitoring of the motor cortex and subcortical pyramidal tract

ABBREVIATIONS CST = corticospinal tract; DTI = diffusion tensor imaging; EMG = electromyogram; MEP = motor evoked potential; MRC = Medical Research Council; $\mathrm{ROI}=$ region of interest.

SUBMITTED June 10,2014. ACCEPTED October 30, 2014.

INCLUDE WHEN CITING Published online June 5, 2015; DOI: 10.3171/2014.10.JNS141289.

DISCLOSURE The authors report no conflict of interest concerning the materials or methods used in this study or the findings specified in this paper. The study was completely financed by the Department of Neurosurgery of the Technical University of Munich. 
has become a pivotal technique for surgery of motor-eloquent lesions. . $19,20,28,31,41,46,48$ These multiple mapping methods aim to identify cortical and subcortical structures of the motor pathway, while continuous monitoring should alert one to impending damage to motor-eloquent areas. Mapping and monitoring together can identify and control surgical corridors for resection and define the limits of tumor resection during surgery for tumors of the rolandic region or those adjacent to the pyramidal tract. $8,12,22,23$, 29,30,41,43,45,46,48

For subcortical motor evoked potential (MEP) mapping, monopolar stimulation has further improved the precision in neurosurgery and has helped to define so-called "safety margins" by which functional boundaries are defined. Electrical stimulation of white matter at the resection cavity is helpful in assessing the proximity to the CST and determining whether the resection should be stopped to avoid injury to the CST. . $^{17,25,26,41,43,46}$

Until recently, the interpretation of this mapping method was 2-fold. Upon eliciting MEPs with a constant subcortical stimulation intensity, one is "very near" to the CST, and new postoperative neurological deficits are to be encountered with a higher probability in those patients in whom a response could be elicited, such as in patients in whom the subcortical stimulation failed to elicit an MEP response. Nevertheless, there have been recent attempts to quantify these distances partially by defining the location of the CST using diffusion tensor imaging (DTI) fiber-tracking tractography. $1,2,4,15,17,25$ However, during surgery, DTI fiber tracking-based visualization of the CST in the navigation system can become inaccurate with the advancing resection of a lesion due to increasing brain shift. To solve this problem, intraoperative DTI fiber tracking was implemented in combination with subcortical stimulation to counter brain shift and to better estimate the proximity to the CST during surgery. ${ }^{35-37,40}$ Other investigators have estimated the stimulation points on postoperative MRI ${ }^{34}$ Furthermore, in a recent study, intraoperative ultrasonography was used to update the navigational imaging to compensate for brain shift and to better estimate proximity to the CST. ${ }^{33}$ These authors described a linear correlation between the subcortical stimulation threshold and the distance to the CST at which each $1 \mathrm{~mA}$ of stimulation threshold corresponded to approximately $1 \mathrm{~mm}$ of distance of the stimulation point to the CST. However, these studies were all limited by the fact that the exact spot within the resection cavity at which subcortical stimulation was performed could not be defined precisely at the time of measurement. Furthermore, the expansion of an electrical field in a 3D space cannot result in a linear relation of stimulation threshold and distance to the CST. Thus, the present study was designed to provide more detailed data on the relationship between the subcortical stimulation threshold and the distance of the stimulation point to the CST.

\section{Methods \\ Patient Cohort}

Between May 2010 and July 2012, patients with lesions close to the subcortical CST and scheduled for surgery were identified and asked to participate in this study. Written informed consent was obtained from all patients who agreed to participate. The institutional review board/ethics committee of the medical faculty of the Technical University of Munich approved all study protocols.

All patients included in the study underwent resection of their lesion, including the part in proximity to the subcortical motor fibers. Intraoperative continuous MEP monitoring by cortical stimulation was performed in all cases, supplemented by subcortical motor stimulation as needed in all cases. An image-guidance system was used for all cases (VectorVision, Brainlab). ${ }^{1}$

\section{Clinical Assessment}

A neurological examination was obtained from every patient preoperatively, directly after surgery, at discharge (7-10 days after surgery), and during follow-up (3-6 months after surgery). Motor function was graded according to the British Medical Research Council (MRC) Scale. Any new surgery-related motor deficit was differentiated as permanent or temporary. A new permanent paresis was defined as a new or aggravated motor deficit due to surgery that did not return to the preoperative status during a follow-up interval of 3 months. A temporary deficit was present when a new or aggravated postoperative paresis resolved within the regular follow-up interval of 3 months.

\section{MRI and DTI Protocol}

Magnetic resonance imaging was acquired before and within 72 hours after surgery in all cases. Most postoperative scans (34/36) were acquired within 48 hours postoperatively. A whole-body 3-T imaging system (Philips Achieva, Philips Electronics) with an 8-channel head coil was used. Three-dimensional imaging was performed by obtaining continuous sagittal images using a T1-weighted $3 \mathrm{D}$ gradient-echo sequence with isotropic voxels of $1 \mathrm{~mm}$ in length after intravenous administration of $0.1 \mathrm{mmol} / \mathrm{kg}$ of gadopentetate dimeglumine. Moreover, DTI sequences were performed with single-shot spin echo planar imaging $(\mathrm{TR} / \mathrm{TE}=7571 / 55 \mathrm{msec}, \mathrm{b}$ values $=0$ and 800 , with 6 orthogonal diffusion directions). Using parallel imaging (sensitivity encoding factor 2), two averages of 73 contiguous 2-mm slices with a matrix of $112 \times 112 \mathrm{~mm}$ covering the whole brain were acquired within 2 minutes and 15 seconds. The DTI data were then interpolated to a matrix of $224 \times 224$, resulting in a voxel size of $0.88 \times 0.88 \times 2$ $\mathrm{mm}^{3}$. In addition, data were corrected for motion artifacts by using the software installed on the scanner. For navigation, a 3D fast-field echo sequence was chosen $(\mathrm{TR} / \mathrm{TE}=$ $9 / 4 \mathrm{msec}$, flip angle $8^{\circ}$ ). A sense factor of 1.5 and a turbo factor of 164 allowed the covering of the whole head in an isotropic resolution of $1 \mathrm{~mm}^{3}$. The 3D data set was then transferred to a BrainLAB iPlan Net server (BrainLab) using the DICOM standard.

\section{Neuronavigation and Fiber Tractography}

The 3D data sets were transferred to the neuronavigation planning system (Brainlab iPlan Cranial 3.0.1, Brainlab AG) and fused with T2-weighted FLAIR and DTI data. The white matter tracts were computed from the DTI data 
set as previously described using Brainlab iPlan Cranial (version 3.0.1). ${ }^{4}$ Seeding was performed using 2 regions of interest (ROIs) for each fiber tracking. One ROI was set to the ipsilateral brainstem at the level of the tentorium; the second ROI was placed over the ipsilateral primary motor cortex. The fractional anisotropy threshold and minimum fiber length were individually defined for each patient, remained constant for each patient, and were all below 0.2 and around $80 \mathrm{~mm}$, respectively. The iteration was started and continued until certain halt criteria were reached, such as fractional anisotropy value and fiber angulation $\left(>30^{\circ}\right)$. Thereafter, the tracked fibers were generated and included in the imaging data set used for image guidance during tumor resection.

\section{Intraoperative Neurophysiological Monitoring}

Anesthesia was induced and maintained by continuous propofol administration while intraoperative analgesia was achieved with continuous administration of remifentanil. Use of a muscle relaxant was avoided during surgery and only used for intubation using rocuronium.

After a craniotomy and durotomy, the central sulcus was identified by phase reversal of the cortical somatosensory evoked potentials ${ }^{5}$ using an 8-channel strip electrode (Inomed Medizintechnik). Cortical MEP monitoring was performed by using 1 of the contacts of the strip electrode as the anode, whereas a subdermal electrode (Inomed needle electrode, Inomed Medizintechnik) positioned above the nasion at Fpz according to the 10-20 International System was used as the opposite pole. For stimulation, square-wave pulses with a duration of $400 \mu \mathrm{sec}$, frequency of $500 \mathrm{~Hz}$, and trains of 5 pulses were applied to the motor cortex. ${ }^{23,51}$ Stimulation intensity ranged from 14 to $26 \mathrm{~mA}$. Electromyograms (EMGs) of 5 different muscles were recorded to monitor MEPs. EMGs were obtained by disposable subdermal needle electrodes (Inomed needle electrode, Inomed Medizintechnik), placed at a distance of approximately $15 \mathrm{~mm}$ from each other, over relevant muscles of the contralateral side of the tumor in a standardized fashion. The muscles recorded were thenar, hypothenar, flexor carpi radialis, and brachial biceps for the upper extremity, and anterior tibial for the lower extremity. Processing of the acquired data was achieved by the Inomed ISIS IOM system (Inomed Medizintechnik). During tumor resection MEPs were elicited every 1-15 seconds depending on the location of resection.

\section{Subcortical Stimulation}

Whenever we believed it to be necessary during the resection, monopolar subcortical MEP stimulation was applied along the border of the resection cavity using a concentric monopolar handheld probe (Inomed Medizintechnik) to identify the proximity of the stimulation point to the CST. For initial subcortical mapping the stimulus conditions were as follows: cathodal stimulation, squarewave pulses with a duration of $0.3 \mathrm{msec}$, frequency of 500 $\mathrm{Hz}$, and a train of 5 pulses were applied, and current intensities varied from 3 to $30 \mathrm{~mA}$. After conclusion of the tumor resection, the point of lowest current intensity still eliciting an MEP response was identified. Thereafter, the stimulation probe was fixed to this point by mounting it to a standard brain retractor to avoid probe movement and ensuring stimulation of the same point during subsequent modification of stimulation parameters (Fig. 1). Subcortical mapping was then modified using either anodal or cathodal stimulation, with pulse durations of $0.3,0.5$, and $0.7 \mathrm{msec}$. With each stimulation modus the MEP threshold was determined, starting with $30 \mathrm{~mA}$ stimulation intensity followed by successive current intensity. The lowest current intensity still eliciting an MEP with each of the 6 different stimulation paradigms (anodal vs cathodal, and 0.3, 0.5 , and $0.7 \mathrm{msec}$ ) was recorded, resulting in 6 measured current intensities as MEP thresholds. Thereafter, the point at which the probe was positioned was marked with a 2.5$\mathrm{mm}$ titanium clip (Weck Hemoclip, Teleflex Medical) to identify the point in postoperative MRI.

\section{Correlation of Subcortical Stimulation With Distance to the CST}

The minimum distance was measured between the subcortical MEP stimulation point, as marked by the titanium clip, and the CST, as depicted by postoperative DTI fiber tracking (Fig. 2). The distances (in $\mathrm{mm}$ ) were correlated to the measured MEP thresholds.

\section{Statistical Analyses}

Statistical analyses were performed using SPSS for Apple (version 2.0, SPSS Inc.). Correlations were analyzed using a regression model, with a probability value $\leq 0.05$ considered significant.

The obtained stimulation setups were plotted as threshold of the stimulation intensity (in $\mathrm{mA}$ ) with 3 different

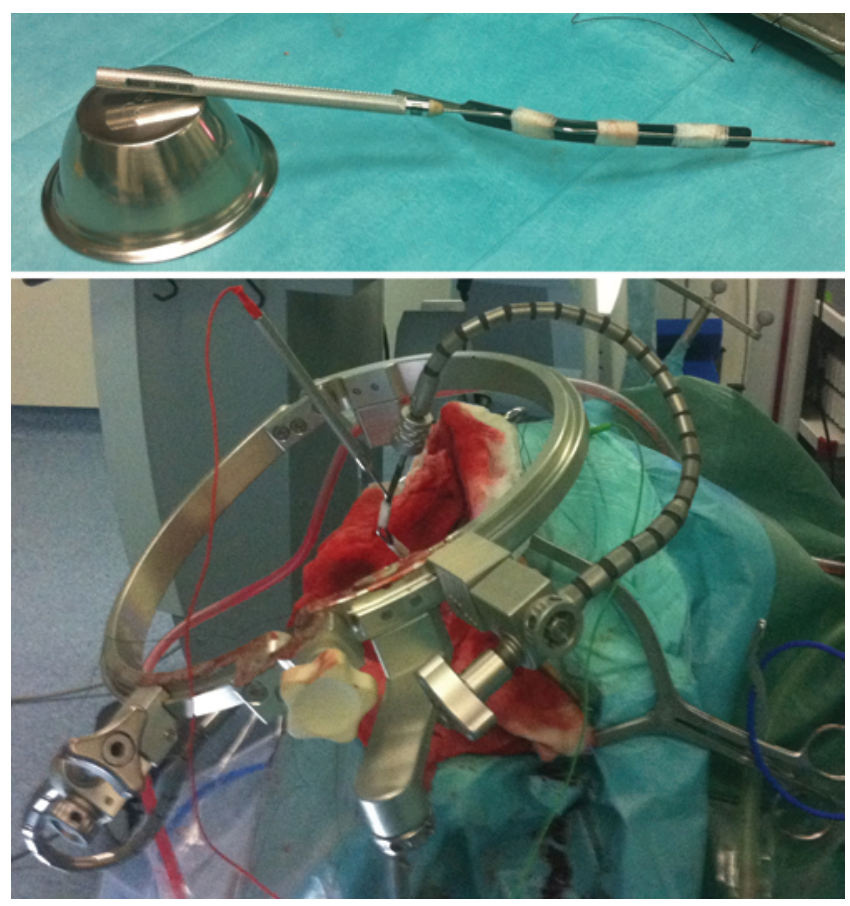

FIG. 1. Monopolar stimulation probe used for subcortical mapping (upper). The probe is mounted onto a standard self-retaining brain retractor system (lower). Figure is available in color online only. 

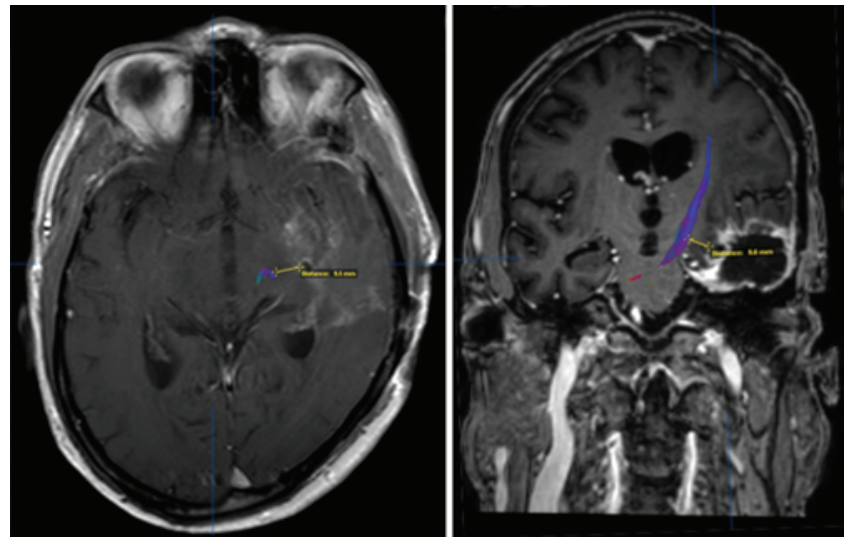

FIG. 2. Postoperative axial (left) and coronal (right) MR images. The distance between the titanium clip and the CST is measured (yellow line). Figure is available in color online only.

pulse durations $(0.3,0.5$, and $0.7 \mathrm{msec})$ as well as for the electrical charge (in $\mu \mathrm{C}$ ) for both cathodal and anodal stimulation against the distance to the CST on scatterplots.

\section{Results}

\section{Theoretical Approach}

The strength of an electrical field at a given distance to an electrical source can be calculated from this equation:

$$
\vec{E}=\frac{Q}{4 \pi \varepsilon} \times \frac{1}{r^{2}}
$$

in which $\vec{E}$ is the strength of the electrical field, $Q$ is the electrical charge, $\varepsilon$ is the electrical constant in a vacuum, and $r$ the distance to the electrical source.

Presuming idealized conditions (i.e., a vacuum, a constant tissue resistance, a spherical source of electrical charge, and a radial expansion of the electrical field), the strength of the electrical field at a given distance to the source according to this equation would decline by $1 / r^{2}$. This means there is no linear relation of the distance of stimulation to the MEP threshold. Therefore, the decline of the electrical field strength would follow an exponential function; the increase in electrical stimulation intensity to achieve the same electrical field strength with increasing distance follows an exponential function as well as the distance dependent on $Q$.

According to the equation above,

$$
r=\sqrt{\frac{Q}{4 \pi \varepsilon \vec{E}}}
$$

where $4 \pi \varepsilon \vec{E}$ is constant, leading to $r=\tilde{c} \times \sqrt{Q}=\tilde{c} \times Q^{b}$.

\section{Patient Characteristics}

Thirty-seven patients (10 women, 27 men) with lesions in motor-eloquent locations underwent resection during this study. The median age of patients at the time of surgery was 53 years (range $21-80$ years).
There were 25 high-grade gliomas (3 WHO Grade III and 22 WHO Grade IV), 5 low-grade gliomas (WHO Grade II), 5 metastases, and 2 arteriovenous malformations (Spetzler-Martin Grade II). Symptoms at presentation were hemiparesis in 12, seizures in 10 (7 generalized and 3 focal), headaches in 3, sensory disturbances in 4, and aphasia in 4 patients. Another 4 patients had an asymptomatic tumor recurrence on MRI. The median preoperative Karnofsky Performance Scale score was 90 (range 70-100).

\section{Surgical Results}

Tumor resection was performed according to microsurgical standards. Gross-total resection was possible and achieved in $75 \%$ of cases $(27 / 36)$ and subtotal resection in $25 \%$ of cases (9/36). One patient was excluded from the study because of postoperative bleeding and displacement of the titanium clip. Upon any instability of MEP signals, amplitude decline, or loss from continuous cortical stimulation, resection was halted, spatulas were removed, and the surgical field was irrigated with warm Ringer's solution. After renormalization/stabilization of MEPs, resection was continued in all but 1 case.

Resection boundaries were determined with the use of subcortical MEP mapping. The resection was stopped if subcortical stimulation in the resection cavity elicited an MEP response at a cathodal stimulation intensity of $3 \mathrm{~mA}$ with 0.3 -msec pulse duration.

\section{Correlation of Subcortical Stimulation Threshold and Distance to the CST}

Subcortical stimulation was successful in all 37 patients, and clips were placed at a stimulation point. One patient showed a displacement of the titanium clip due to a postoperative secondary hemorrhage and therefore was excluded from further analysis. In 35 patients 1 titanium clip was used as a marker, and in 1 patient we stimulated 2 different points that were marked with 2 titanium clips. The anodal stimulation intensity was 3-30 mA, and the resulting stimulation charge (stimulation intensity $\times$ pulse duration) was 0.9-11.9 $\mu \mathrm{C}$. The cathodal stimulation intensity was 3-17 $\mathrm{mA}$, and the stimulation charge was $0.9-$ $9.8 \mu \mathrm{C}$. The measured distances between the CST and the titanium clip ranged from 2.6 to $21.9 \mathrm{~mm}$.

The obtained MEP stimulation thresholds were plotted on scatterplots as thresholds of anodal or cathodal stimulation intensity with different pulse durations (Fig. 3) and as anodal or cathodal stimulation charge (Fig. 4) against the measured distance to the CST as measured on MR images.

The relationship between the distance from the CST to the titanium clip and the cathodal or anodal stimulation was assumed to be of the functional form $Q=\alpha * r^{\beta}$, in which $Q$ is stimulation intensity (electrical charge) and $r$ is the distance to the $\mathrm{CST},{ }^{27}$ or

$$
r=\sqrt[\beta]{\frac{Q}{\alpha}} .
$$

The multiplicative parameter $\alpha$ and the exponential (exp) parameter $\beta$ were derived from a linear regression model using the logarithm of stimulation as the dependent variable and the logarithm of distance as the independent 

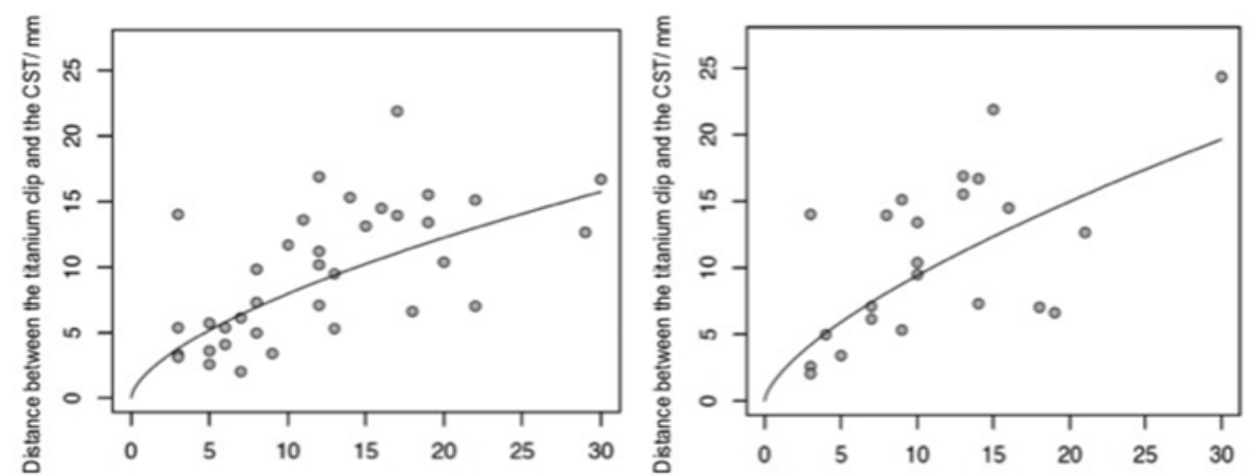

Anodal stimulation with $0.3 \mathrm{~ms}$ pulse duration / $\mathrm{mA}$

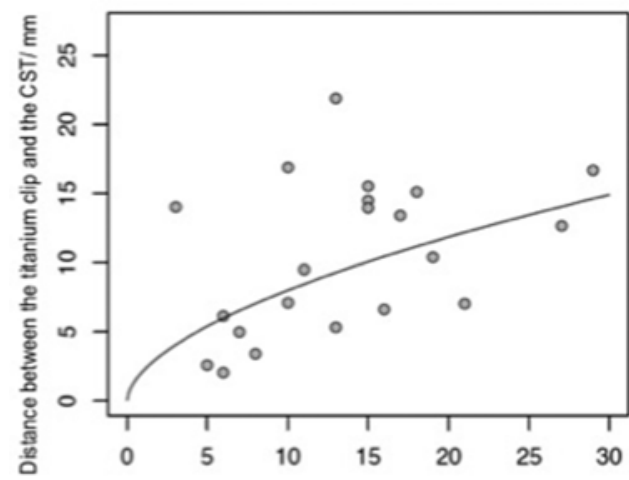

Cathodal stimulation with $0.3 \mathrm{~ms}$ Dulse duration/ $\mathrm{mA}$

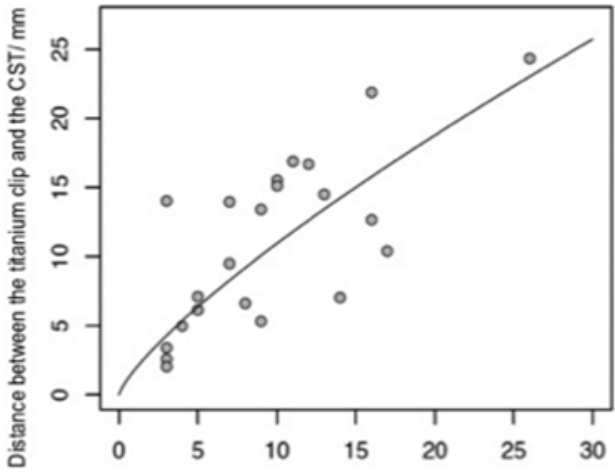

Anodal stimulation with $0.5 \mathrm{~ms}$ pulse duration $/ \mathrm{mA}$

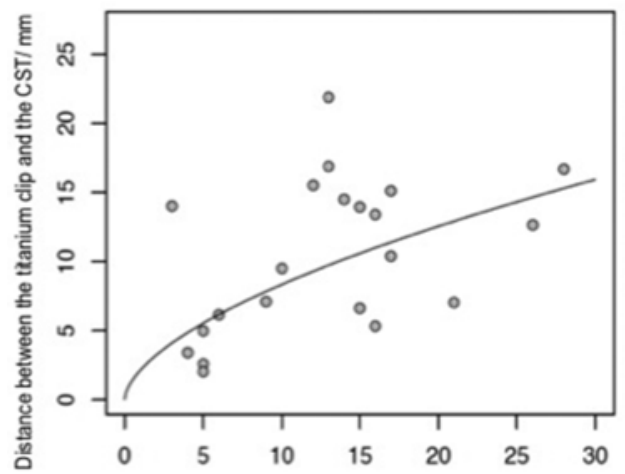

Cathodal stimulation with $0.5 \mathrm{~ms}$ pulse duration/ $\mathrm{mA}$

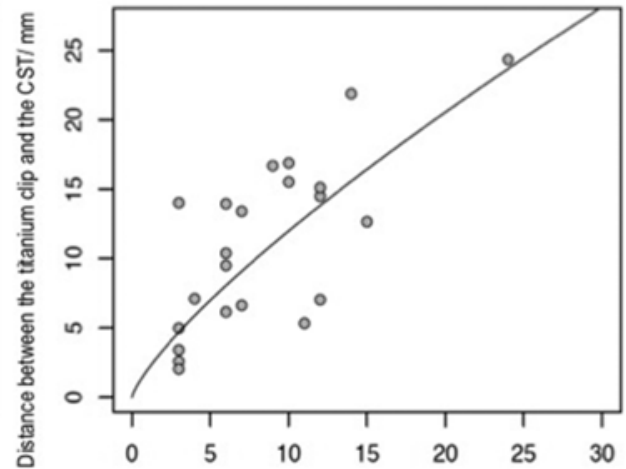

Anodal stimulation with $0.7 \mathrm{~ms}$ pulse duration / $\mathrm{mA}$

Cathodal stimulation with $0.7 \mathrm{~ms}$ pulse duration/ $\mathrm{mA}$

FIG. 3. Scatterplots of the proximity correlations between the stimulation intensity $(\mathrm{mA})$, with different pulse durations $(0.3,0.5$, and $0.7 \mathrm{msec}$ ) using anodal or cathodal stimulation, and the distance to the CST.

variable, following the rules of logarithms. Regression analysis revealed a nonlinear correlation for both anodal and cathodal stimulation. For anodal stimulation intensity with $0.3-, 0.5-$, and $0.7-\mathrm{msec}$ pulse duration, and using the complete charge in coulombs, respectively, the following results were obtained: $0.3 \mathrm{msec}$, distance $=\exp (0.653) \times$ intensity ${ }^{0.619}\left(\mathrm{R}^{2}=0.419, \mathrm{p}<0.001 ; \mathrm{r}=0.651, \mathrm{p}<0.001\right) ; 0.5$ msec, distance $=\exp (0.778) \times$ intensity $^{0.566}\left(\mathrm{R}^{2}=0.243, \mathrm{p}=\right.$ $0.023 ; \mathrm{r}=0.437, \mathrm{p}=0.047) ; 0.7 \mathrm{msec}$, distance $=\exp (0.766)$ $\times$ intensity ${ }^{0.589}\left(R^{2}=0.316, p=0.008 ; r=0.41, p=0.065\right)$; and distance $=\exp (1.443) \times$ intensity ${ }^{0.449}\left(\mathrm{R}^{2}=0.272, \mathrm{p}<\right.$ $0.001 ; \mathrm{r}=0.518, \mathrm{p}<0.001)$ for the charge in coulombs.

For cathodal stimulation intensity with $0.3-, 0.5-$, and $0.7-\mathrm{msec}$ pulse duration, and using the complete charge in coulombs, respectively, the following results were obtained: $0.3 \mathrm{msec}$, distance $=\exp (0.703) \times$ intensity $\mathrm{y}^{0.669}\left(\mathrm{R}^{2}=\right.$ $0.42, \mathrm{p}=0.001 ; \mathrm{r}=0.548, \mathrm{p}=0.007) ; 0.5 \mathrm{msec}$, distance $=$ $\exp (0.601) \times$ intensity ${ }^{0.778}\left(\mathrm{R}^{2}=0.538, \mathrm{p}<0.001 ; \mathrm{r}=0.672\right.$, $\mathrm{p}=0.001) ; 0.7 \mathrm{msec}$, distance $=\exp (0.694) \times$ intensity ${ }^{0.778}$ $\left(\mathrm{R}^{2}=0.494, \mathrm{p}<0.001 ; \mathrm{r}=0.65, \mathrm{p}=0.001\right)$; and distance $=$ $\exp (1.341) \times$ intensity ${ }^{0.652}\left(\mathrm{R}^{2}=0.426, \mathrm{p}<0.001 ; \mathrm{r}=0.607, \mathrm{p}\right.$ $<0.001$ ) for the charge in coulombs (Table 1, Figs. 3 and 4).

\section{Correlation of Electrophysiological Findings to Postoperative Clinical Outcome}

Cortical MEP monitoring was possible in all patients. Mean cortical stimulation intensity was $23 \mathrm{~mA}$ (range 

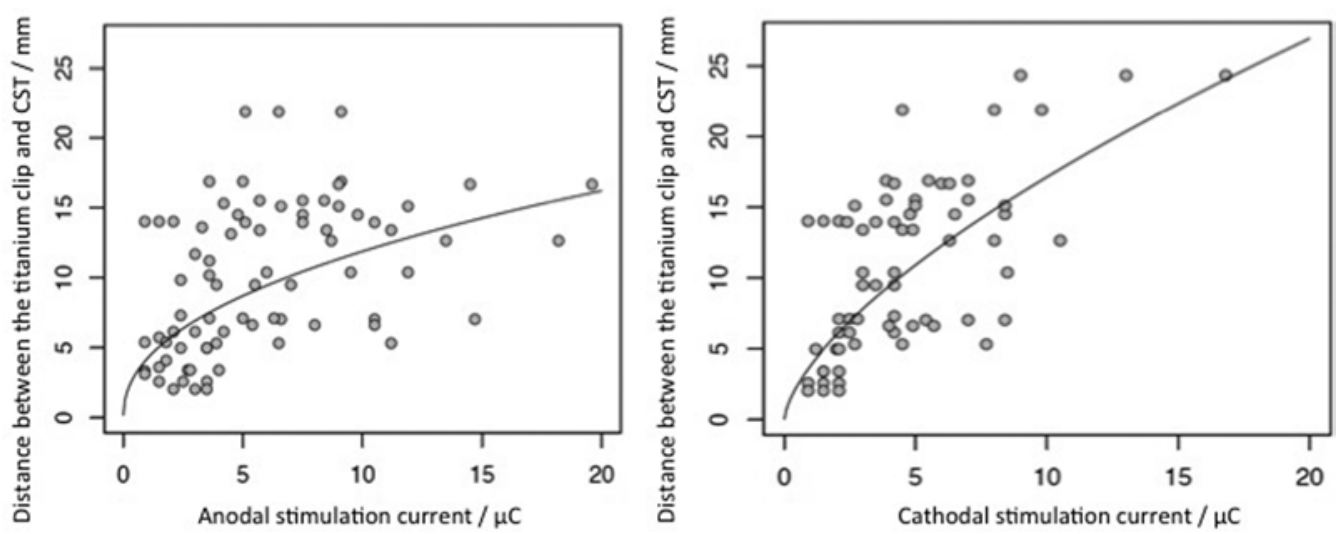

FIG. 4. Scatterplots of the proximity correlations between the current intensity $(\mathrm{mA} \times \mu \mathrm{sec}=\mu \mathrm{C})$, using anodal (left) or cathodal (right) stimulation, and the distance to the CST.

16-27 mA). MEPs were stable in 83\% (30/36) of cases according to the intraoperative impression of the neurophysiologist. In 14\% (5/36) of all cases, MEPs were unstable and dropped more than 50\% of baseline MEP amplitude but recovered in almost all cases. There was 1 case of an irreversible MEP loss during the final stages of resection of an insular glioma; however, this was at the end of resection and monitoring was terminated 5 minutes later, so MEP recovery cannot be excluded. Resection was not terminated because subcortical stimulation illustrated that the CST was still intact. This patient experienced a transient hemiplegia that resolved rapidly within 2 days. Postoperatively, 14\% (5/36) had a new temporary motor deficit (all with $\leq 5-\mathrm{mm}$ distance from the CST), but there were no cases of new permanent paresis.

\section{Discussion}

During resection of motor-eloquent lesions, subcortical MEP mapping is an excellent intraoperative method to determine the distance to the CST, and the risk of direct damage to the CST is markedly reduced using this meth- od. Cathodal stimulation is more appropriate for subcortical MEP mapping as it requires lower stimulation intensities and provides better tissue penetration compared with anodal stimulation. Anodal stimulation at $0.5 \mathrm{msec}$ and $0.7 \mathrm{msec}$ did not show a significant correlation between stimulation threshold and distance to the CST. Furthermore, the present data show a nonlinear correlation between stimulation threshold and the distance to the CST. A stimulation with cathodal polarity and a duration of 0.5 msec or 0.7 msec appears to be most viable because the resulting curves are closest to the simple rule that $1 \mathrm{~mA}$ of subcortical stimulation intensity to elicit an MEP response resembles 1-mm distance of the stimulation point to the CST.

For the last 20 years, increasing evidence in the literature has revealed that a more extensive resection of lowgrade gliomas is associated with a higher life expectancy and a reduced risk of malignant transformation.,10,14,42,44 Likewise, in high-grade gliomas, a complete resection can improve overall survival, while the role of a subtotal resection is an issue of ongoing debate. . $21,45,47$ In conclusion, the aim of surgical treatment of gliomas is to achieve the

TABLE 1. Correlation between subcortical stimulation threshold and distance to the CST

\begin{tabular}{|c|c|c|c|c|c|c|}
\hline \multirow[b]{2}{*}{ Stimulation Parameter } & \multicolumn{4}{|c|}{ Linear Regression Model ${ }^{*}$} & \multicolumn{2}{|c|}{ Spearman Correlation $\dagger$} \\
\hline & a & $b$ & $\mathrm{R}^{2}$ & $p$ Value & rho & p Value \\
\hline Anodal stimulation w/ $0.3 \mathrm{msec}$ & 0.653 & 0.619 & 0.419 & $<0.001$ & 0.651 & $<0.001$ \\
\hline Cathodal stimulation w/ $0.3 \mathrm{msec}$ & 0.703 & 0.669 & 0.42 & 0.001 & 0.548 & 0.007 \\
\hline Anodal stimulation w/ $0.5 \mathrm{msec}$ & 0.778 & 0.566 & 0.243 & 0.023 & 0.437 & 0.047 \\
\hline Cathodal stimulation w/ $0.5 \mathrm{msec}$ & 0.601 & 0.778 & 0.538 & $<0.001$ & 0.672 & 0.001 \\
\hline Anodal stimulation w/ $0.7 \mathrm{msec}$ & 0.766 & 0.589 & 0.316 & 0.008 & 0.41 & 0.065 \\
\hline Cathodal stimulation w/ $0.7 \mathrm{msec}$ & 0.694 & 0.778 & 0.494 & $<0.001$ & 0.65 & 0.001 \\
\hline Anodal current $w / \mu \mathrm{C}$ & 1.443 & 0.449 & 0.272 & $<0.001$ & 0.518 & $<0.001$ \\
\hline Cathodal current $w / \mu C$ & 1.341 & 0.652 & 0.426 & $<0.001$ & 0.607 & $<0.001$ \\
\hline
\end{tabular}

* The multiplicative parameter "a" and the exponential parameter "b" were derived from a linear regression model using the logarithm of stimulation as dependent variables and the logarithm of distance as independent variables following the rules of logarithms, according to the equation: distance $=\exp (a) \times$ stimulation $^{b}$.

$\dagger$ The Spearman correlation was applied for the different stimulations, and the measured distances between the CST and titanium clips were calculated. 
maximum possible extent of resection, but without the induction of surgery-related permanent deficits. To achieve this goal, recent data show that a multimodal setup including preoperative mapping, DTI fiber tracking, and intraoperative monitoring can reduce the risk of neurological damage. $9,35,36,40$

DTI fiber tracking can improve the extent of resection and prevent unintentional resection of adjacent subcortical pathways. ${ }^{50,52} \mathrm{Wu}$ et al. reported on 238 patients with gliomas who were randomized to DTI imaging versus traditional MRI neuronavigation without DTI, in whom postoperative motor deterioration occurred in $38.2 \%$ of control cases and $15.3 \%$ of the study cases. ${ }^{52}$ However, one major problem of this approach-navigation of tracked fibers-is brain shift during surgery, which is affected by CSF loss, tumor resection, surgical retraction, and gravity. Nimsky et al. ${ }^{32}$ have clearly shown in 37 patients that the white matter tracts shift at a range from -8 to $15 \mathrm{~mm}$ (mean 2.7 $\pm 6.0 \mathrm{~mm}$ ). Shifting was observed in an inward or outward direction in $29.7 \%$ and $62.2 \%$, respectively. The authors concluded that "this shifting emphasizes the need for an intraoperative update of navigation systems during resection of deep-seated tumor portions near eloquent brain areas." Therefore, to rely on navigated fiber tracks to preserve motor function is an unreliable technique prone to errors.

To that end, subcortical stimulation helps better define the proximity of a certain point of resection to the CST during surgery and therefore helps neurosurgeons to perform safer and more radical tumor resections in the vicinity of the CST. ${ }^{11,12,17}$ Subcortical stimulation is the most reliable method to estimate the proximity to the CST during resection of deep-seated lesions in the white matter. ${ }^{3}$ So far, some reports have combined the use of subcortical stimulation with neuronavigation and DTI fiber tracking and have analyzed the distances between stimulation points and the CST to clearly define the relationship between stimulation threshold and distance to the CST. 2,3,15,18,26,40,53 However, different stimulation conditions were used, resulting in slightly different results.

Berman et al. ${ }^{3}$ used $60-\mathrm{Hz}$ bipolar stimulation in 9 patients. The calculated distance from the CST was $8.7 \pm 3.1$ $\mathrm{mm}$ in this study, with stimulation intensities ranging from 8 to $12 \mathrm{~mA}$. Kamada et al. ${ }^{16}$ used a "train of five" monopolar cathodal stimulation in 6 patients. They found a nonlinear correlation between the stimulus intensity and the distance to the CST by using a convergent calculation. This calculation suggested that the minimum stimulus intensities with $20,15,10$, and $5 \mathrm{~mA}$ indicated that the stimulus points were approximately $16,13.2,8.6$, and $4.8 \mathrm{~mm}$ from the CST, respectively. The convergent calculation formulated $1.8 \mathrm{~mA}$ as the electrical threshold of the CST. Mikuni et al. ${ }^{26}$ used $50-\mathrm{Hz}$ bipolar stimulation in 22 patients. MEPs were consistently elicited at distances less than 7 $\mathrm{mm}$ (6 patients) from the CST, but were consistently absent at distances more than $13 \mathrm{~mm}$ (7 patients) from the CST. However, corresponding stimulation intensity was not noted. Ohue et al. ${ }^{34}$ used a "train of five" bipolar cathodal stimulation in 32 patients. They found a significant linear correlation between stimulation intensity and the distance from the CST. Their measurements showed that minimum stimulation intensity with $5,10,15$, and $20 \mathrm{~mA}$ indicated that the stimulus points were 5.0, 10.2, 15.3, and $20.5 \mathrm{~mm}$ from the CST. All these studies used preoperative MRI navigation to estimate the distance of the stimulation point to the CST. However, as previously mentioned, brain shift is a significant problem in the use of preoperative MRI to estimate the distance to the CST during surgery, leading to potential data inaccuracies.

To minimize these inaccuracies, others have measured the distance between the subcortical stimulation points and the CST using intraoperative MRI with DTI fiber tracking. ${ }^{35,40}$ Ozawa et al. ${ }^{35}$ used bipolar stimulation in 7 patients. The distance from the stimulation point to the CST was 0-4.7 $\mathrm{mm}$ (mean $1.4 \pm 2.1 \mathrm{~mm}$ ) with stimulation intensity ranging from 4 to $20 \mathrm{~mA}$. When the distance was more than $5 \mathrm{~mm}$, no MEPs could be elicited. Prabhu et al. ${ }^{40}$ used a "train of five" monopolar anodal stimulation in 12 patients. The mean stimulus intensity was $10.4 \pm$ $5.2 \mathrm{~mA}$, and the mean distance from the navigated probe tip to the CST was $7.4 \pm 4.5 \mathrm{~mm}$. However, intraoperative MRI with DTI fiber tracking is less accurate in localizing the CST. In a study by Nossek et al., ${ }^{33}$ intraoperative ultrasonography was used to update the navigation imaging. They used a "train of five" cathodal stimulation in 55 patients. A linear correlation was found between stimulation intensity and distance to the CST, a relationship of $0.97 \mathrm{~mA}$ for every $1 \mathrm{~mm}$ distance from the CST. However, these assumptions are a matter of some debate because distributions of voltage and electrical charge in the brain generally do not show a linear decay, but instead show a nonlinear decay as a function of distance from the stimulation electrode. ${ }^{27}$ Moreover, all of the above-mentioned results differ, not only because of the imaging modality chosen by which the measurements were performed, but also because different stimulation conditions were used. Some reports have used the $50-\mathrm{Hz}$ stimulation technique, which was first described by Penfield in 1937; others have used the multipulse train stimulation technique ("train of five") first described by Taniguchi et al. in 1993. ${ }^{51}$ Stimulation conditions were also different with regard to bipolar versus monopolar stimulation, as well as stimulation polarity (anodal vs cathodal) and stimulation pulse duration. A report by Szelényi et al. ${ }^{49}$ compared different stimulation conditions in 20 patients. Both the multipulse train stimulation and the $50-\mathrm{Hz}$ stimulation techniques, using bipolar or monopolar stimulation probes, were examined. The authors concluded that "subcortical stimulation with a monopolar probe and a multipulse technique is most efficient for the purpose of identifying the CST."

In summary, a comprehensive analysis of the currently available literature showed no reliable method for estimating distance to the CST during resection of lesions in motor-eloquent locations because of the different stimulation conditions and analysis methods used thus far.

The aim of our study was to examine different stimulation conditions using monopolar multipulse stimulation to better estimate the proximity to the CST during surgery with an up-to-date setup. We were successful in obtaining MEPs in all patients. None of the patients had permanent new neurological deficits, regardless of the measured proximity to the CST. Subcortical MEP mapping 

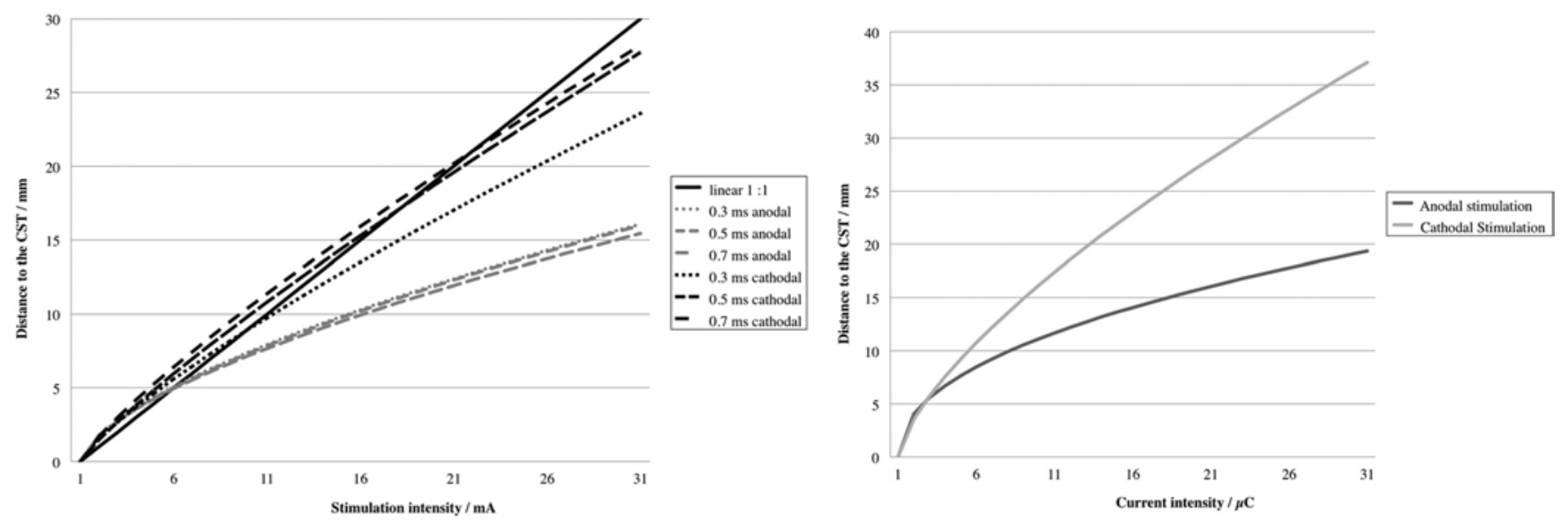

FIG. 5. Proximity correlation graph of the estimated distance to the CST upon subcortical MEP stimulation using 0.3-, 0.5-, and $0.7-\mathrm{msec}$ pulse duration with either anodal or cathodal stimulation according to the calculated regression parameters in Table 1 (left), and proximity correlation graph of the estimated current intensities using either anodal or cathodal stimulation to the distance to the CST according to the calculated regression parameters in Table 1 (right).

was more effective with a cathodal current; that is, a lower stimulation intensity was needed to observe a stimulation effect. Moreover, distance to the CST and anodal stimulation intensity with 0.5 - and $0.7-\mathrm{msec}$ pulse durations were not statistically significant (Table 1). There was a nonlinear correlation between stimulation current and the distance to the CST, in which the CST was reached sooner than expected according to a linear correlation pattern, as reported frequently in the past (Fig. 5). Therefore subcortical stimulation should be used more frequently, especially during the final stages of tumor resection close to the CST.

Regarding limitations of the present approach, it could be argued that, during the time span between surgery and postoperative imaging, spatial variations could occur by postoperative swelling or hematoma. This cannot be ruled out completely. However, most patients underwent postoperative imaging within 48 hours and had postoperative dexamethasone to avoid tissue swelling, thereby reducing the impact of this potential confounding factor. Furthermore, in most cases the remaining tissue volume between the resection border and CST was low, and as a consequence further swelling would lead only to minor changes of the measured distance. An influence of hematoma can be excluded because none of the patients in the final evaluation showed a postoperative hematoma that led to any spatial disturbances. Additionally, DTI fiber tracking is potentially prone to operator-associated variations, which is minimized by a limitation to 1 observer and a reproducible fiber-tracking method. Therefore, major confounders are excluded.

\section{Conclusions}

Subcortical stimulation is an excellent intraoperative method to determine the distance to the CST during resection of lesions in motor-eloquent locations and allows one to minimize the risk of harming the CST. Cathodal stimulation appears more adequate for subcortical stimulation because it requires lower stimulation intensities. There is a nonlinear correlation between stimulation current and the distance to the CST; thus one is approaching the CST sooner than expected following a linear correlation pattern, as reported frequently in the past. Therefore, subcortical stimulation should be used more frequently during tumor resection close to the CST.

\section{References}

1. Bello L, Castellano A, Fava E, Casaceli G, Riva M, Scotti $\mathrm{G}$, et al: Intraoperative use of diffusion tensor imaging fiber tractography and subcortical mapping for resection of gliomas: technical considerations. Neurosurg Focus 28(2):E6, 2010

2. Bello L, Gambini A, Castellano A, Carrabba G, Acerbi F, Fava E, et al: Motor and language DTI fiber tracking combined with intraoperative subcortical mapping for surgical removal of gliomas. Neuroimage 39:369-382, 2008

3. Berman JI, Berger MS, Chung SW, Nagarajan SS, Henry RG: Accuracy of diffusion tensor magnetic resonance imaging tractography assessed using intraoperative subcortical stimulation mapping and magnetic source imaging. J Neurosurg 107:488-494, 2007

4. Buchmann N, Gempt J, Stoffel M, Foerschler A, Meyer B, Ringel F: Utility of diffusion tensor-imaged (DTI) motor fiber tracking for the resection of intracranial tumors near the corticospinal tract. Acta Neurochir (Wien) 153:68-74, 2011

5. Cedzich C, Taniguchi M, Schäfer S, Schramm J: Somatosensory evoked potential phase reversal and direct motor cortex stimulation during surgery in and around the central region. Neurosurgery 38:962-970, 1996

6. Chaichana KL, Zadnik P, Weingart JD, Olivi A, Gallia GL, Blakeley J, et al: Multiple resections for patients with glioblastoma: prolonging survival. J Neurosurg 118:812-820, 2013

7. Chang EF, Clark A, Smith JS, Polley MY, Chang SM, Barbaro NM, et al: Functional mapping-guided resection of low-grade gliomas in eloquent areas of the brain: improvement of long-term survival. Clinical article. J Neurosurg 114:566-573, 2011

8. Deletis V: Intraoperative monitoring of the functional integrity of the motor pathways. Adv Neurol 63:201-214, 1993

9. De Witt Hamer PC, Robles SG, Zwinderman AH, Duffau H, 
Berger MS: Impact of intraoperative stimulation brain mapping on glioma surgery outcome: a meta-analysis. J Clin Oncol 30:2559-2565, 2012

10. Duffau H: Lessons from brain mapping in surgery for lowgrade glioma: insights into associations between tumour and brain plasticity. Lancet Neurol 4:476-486, 2005

11. Duffau H, Capelle L, Denvil D, Sichez N, Gatignol P, Taillandier L, et al: Usefulness of intraoperative electrical subcortical mapping during surgery for low-grade gliomas located within eloquent brain regions: functional results in a consecutive series of 103 patients. J Neurosurg 98:764-778, 2003

12. Duffau H, Lopes M, Arthuis F, Bitar A, Sichez JP, Van Effenterre R, et al: Contribution of intraoperative electrical stimulations in surgery of low grade gliomas: a comparative study between two series without (1985-96) and with (1996-2003) functional mapping in the same institution. J Neurol Neurosurg Psychiatry 76:845-851, 2005

13. Fontaine D, Capelle L, Duffau H: Somatotopy of the supplementary motor area: evidence from correlation of the extent of surgical resection with the clinical patterns of deficit. Neurosurgery 50:297-305, 2002

14. Jakola AS, Myrmel KS, Kloster R, Torp SH, Lindal S, Unsgård $\mathrm{G}$, et al: Comparison of a strategy favoring early surgical resection vs a strategy favoring watchful waiting in lowgrade gliomas. JAMA 308:1881-1888, 2012

15. Kamada K, Todo T, Masutani Y, Aoki S, Ino K, Takano T, et al: Combined use of tractography-integrated functional neuronavigation and direct fiber stimulation. J Neurosurg 102:664-672, 2005

16. Kamada K, Todo T, Ota T, Ino K, Masutani Y, Aoki S, et al: The motor-evoked potential threshold evaluated by tractography and electrical stimulation. J Neurosurg 111:785-795, 2009

17. Keles GE, Lundin DA, Lamborn KR, Chang EF, Ojemann G, Berger MS: Intraoperative subcortical stimulation mapping for hemispherical perirolandic gliomas located within or adjacent to the descending motor pathways: evaluation of morbidity and assessment of functional outcome in 294 patients. J Neurosurg 100:369-375, 2004

18. Kinoshita M, Yamada K, Hashimoto N, Kato A, Izumoto S, Baba T, et al: Fiber-tracking does not accurately estimate size of fiber bundle in pathological condition: initial neurosurgical experience using neuronavigation and subcortical white matter stimulation. Neuroimage 25:424-429, 2005

19. Kombos T, Picht T, Derdilopoulos A, Suess O: Impact of intraoperative neurophysiological monitoring on surgery of high-grade gliomas. J Clin Neurophysiol 26:422-425, 2009

20. Kombos T, Suess O, Ciklatekerlio O, Brock M: Monitoring of intraoperative motor evoked potentials to increase the safety of surgery in and around the motor cortex. J Neurosurg 95:608-614, 2001

21. Kreth FW, Thon N, Simon M, Westphal M, Schackert G, Nikkhah G, et al: Gross total but not incomplete resection of glioblastoma prolongs survival in the era of radiochemotherapy. Ann Oncol 24:3117-3123, 2013

22. Krieg SM, Schäffner M, Shiban E, Droese D, Obermüller T, Gempt J, et al: Reliability of intraoperative neurophysiological monitoring using motor evoked potentials during resection of metastases in motor-eloquent brain regions: clinical article. J Neurosurg 118:1269-1278, 2013

23. Krieg SM, Shiban E, Droese D, Gempt J, Buchmann N, Pape $\mathrm{H}$, et al: Predictive value and safety of intraoperative neurophysiological monitoring with motor evoked potentials in glioma surgery. Neurosurgery 70:1060-1071, 2012

24. Laws ER Jr, Taylor WF, Clifton MB, Okazaki H: Neurosurgical management of low-grade astrocytoma of the cerebral hemispheres. J Neurosurg 61:665-673, 1984

25. Mikuni N, Okada T, Enatsu R, Miki Y, Hanakawa T, Uraya- ma S, et al: Clinical impact of integrated functional neuronavigation and subcortical electrical stimulation to preserve motor function during resection of brain tumors. J Neurosurg 106:593-598, 2007

26. Mikuni N, Okada T, Nishida N, Taki J, Enatsu R, Ikeda A, et al: Comparison between motor evoked potential recording and fiber tracking for estimating pyramidal tracts near brain tumors. J Neurosurg 106:128-133, 2007

27. Miocinovic S, Lempka SF, Russo GS, Maks CB, Butson CR, Sakaie KE, et al: Experimental and theoretical characterization of the voltage distribution generated by deep brain stimulation. Exp Neurol 216:166-176, 2009

28. Neuloh G, Pechstein U, Cedzich C, Schramm J: Motor evoked potential monitoring with supratentorial surgery. Neurosurgery 54:1061-1072, 2004

29. Neuloh G, Pechstein U, Schramm J: Motor tract monitoring during insular glioma surgery. J Neurosurg 106:582-592, 2007

30. Neuloh G, Schramm J: Monitoring of motor evoked potentials compared with somatosensory evoked potentials and microvascular Doppler ultrasonography in cerebral aneurysm surgery. J Neurosurg 100:389-399, 2004

31. Neuloh G, Schramm J: What the surgeon wins, and what the surgeon loses from intraoperative neurophysiologic monitoring? Acta Neurochir (Wien) 147:811-813, 2005

32. Nimsky C, Ganslandt O, Hastreiter P, Wang R, Benner T, Sorensen AG, et al: Preoperative and intraoperative diffusion tensor imaging-based fiber tracking in glioma surgery. Neurosurgery 61 (1 Suppl):178-186, 2007

33. Nossek E, Korn A, Shahar T, Kanner AA, Yaffe H, Marcovici $\mathrm{D}$, et al: Intraoperative mapping and monitoring of the corticospinal tracts with neurophysiological assessment and 3 -dimensional ultrasonography-based navigation. Clinical article. J Neurosurg 114:738-746, 2011

34. Ohue S, Kohno S, Inoue A, Yamashita D, Harada H, Kumon Y, et al: Accuracy of diffusion tensor magnetic resonance imaging-based tractography for surgery of gliomas near the pyramidal tract: a significant correlation between subcortical electrical stimulation and postoperative tractography. Neurosurgery 70:283-294, 2012

35. Ozawa N, Muragaki Y, Nakamura R, Hori T, Iseki H: Shift of the pyramidal tract during resection of the intraaxial brain tumors estimated by intraoperative diffusion-weighted imaging. Neurol Med Chir (Tokyo) 49:51-56, 2009

36. Ozawa N, Muragaki Y, Nakamura R, Iseki H: Identification of the pyramidal tract by neuronavigation based on intraoperative diffusion-weighted imaging combined with subcortical stimulation. Stereotact Funct Neurosurg 87:18-24, 2009

37. Ozawa N, Muragaki Y, Nakamura R, Lseki H: Intraoperative diffusion-weighted imaging for visualization of the pyramidal tracts. Part II: clinical study of usefulness and efficacy. Minim Invasive Neurosurg 51:67-71, 2008

38. Penfield W, Boldrey E: Somatic motor and sensory representation in the cerebral cortex of man as studied by electric stimulation. Brain 60:389-443, 1937

39. Polin RS, Marko NF, Ammerman MD, Shaffrey ME, Huang W, Anderson FA Jr, et al: Functional outcomes and survival in patients with high-grade gliomas in dominant and nondominant hemispheres. J Neurosurg 102:276-283, 2005

40. Prabhu SS, Gasco J, Tummala S, Weinberg JS, Rao G: Intraoperative magnetic resonance imaging-guided tractography with integrated monopolar subcortical functional mapping for resection of brain tumors. Clinical article. J Neurosurg 114:719-726, 2011

41. Raabe A, Beck J, Schucht P, Seidel K: Continuous dynamic mapping of the corticospinal tract during surgery of motor eloquent brain tumors: evaluation of a new method. J Neurosurg 120:1015-1024, 2014

42. Sanai N, Berger MS: Glioma extent of resection and its im- 
pact on patient outcome. Neurosurgery 62:753-764, 264266, 2008

43. Sanai N, Berger MS: Intraoperative stimulation techniques for functional pathway preservation and glioma resection. Neurosurg Focus 28(2):E1, 2010

44. Sanai N, Mirzadeh Z, Berger MS: Functional outcome after language mapping for glioma resection. $\mathbf{N}$ Engl J Med 358:18-27, 2008

45. Sanai N, Polley MY, McDermott MW, Parsa AT, Berger MS: An extent of resection threshold for newly diagnosed glioblastomas. J Neurosurg 115:3-8, 2011

46. Seidel K, Beck J, Stieglitz L, Schucht P, Raabe A: The warning-sign hierarchy between quantitative subcortical motor mapping and continuous motor evoked potential monitoring during resection of supratentorial brain tumors. J Neurosurg 118:287-296, 2013

47. Stummer W, Pichlmeier U, Meinel T, Wiestler OD, Zanella F, Reulen HJ: Fluorescence-guided surgery with 5-aminolevulinic acid for resection of malignant glioma: a randomised controlled multicentre phase III trial. Lancet Oncol 7:392401, 2006

48. Szelényi A, Langer D, Kothbauer K, De Camargo AB, Flamm ES, Deletis V: Monitoring of muscle motor evoked potentials during cerebral aneurysm surgery: intraoperative changes and postoperative outcome. J Neurosurg 105:675681,2006

49. Szelényi A, Senft C, Jardan M, Forster MT, Franz K, Seifert $\mathrm{V}$, et al: Intra-operative subcortical electrical stimulation: a comparison of two methods. Clin Neurophysiol 122:14701475, 2011

50. Talos IF, Zou KH, Kikinis R, Jolesz FA: Volumetric assess- ment of tumor infiltration of adjacent white matter based on anatomic MRI and diffusion tensor tractography. Acad Radiol 14:431-436, 2007

51. Taniguchi M, Cedzich C, Schramm J: Modification of cortical stimulation for motor evoked potentials under general anesthesia: technical description. Neurosurgery 32:219-226, 1993

52. Wu JS, Zhou LF, Tang WJ, Mao Y, Hu J, Song YY, et al: Clinical evaluation and follow-up outcome of diffusion tensor imaging-based functional neuronavigation: a prospective, controlled study in patients with gliomas involving pyramidal tracts. Neurosurgery 61:935-949, 2007

53. Yamaguchi F, Takahashi H, Teramoto A: Navigation-assisted subcortical mapping: intraoperative motor tract detection by bipolar needle electrode in combination with neuronavigation system. J Neurooncol 93:121-125, 2009

\section{Author Contributions}

Conception and design: Ringel, Shiban. Acquisition of data: Ringel, Shiban, Krieg, Buchmann, Obermueller. Analysis and interpretation of data: Ringel, Shiban, Boeckh-Behrens. Drafting the article: Ringel, Shiban. Critically revising the article: Ringel, Meyer. Reviewed submitted version of manuscript: Ringel, Wostrack, Meyer. Statistical analysis: Haller.

\section{Correspondence}

Florian Ringel, Department of Neurosurgery, Klinikum rechts der Isar, Technische Universität München, Ismaninger Str. 22, Munich 81675, Germany. email: florian.ringel@lrz.tum.de. 